\title{
Sexual harassment rampant in science and worst in medicine, says report
}

\author{
n Cite as: CMAJ 2018 July 9;190:E842-3. doi: 10.1503/cmaj.109-5625
}

Posted on cmajnews.com on June 20, 2018.

W omen face frequent sexual harassment in science, and particularly in medicine, according to a report by the United States National Academies of Sciences, Engineering and Medicine.

Decades of efforts to curb the abuse have failed, even as more women enter scientific fields. The report calls on universities to overhaul how they prevent and respond to incidents, which could include diffusing the power of senior men, who often control the careers of women below them.

"We really have to move beyond a mindset of legal compliance and liability and think about the ways we can change the climate," said Dr. Paula Johnson, cochair of the committee that produced the report.

Academic workplaces report rates of sexual harassment second only to the military. Studies show more than half of female faculty and staff, and up to half of female students, in scientific fields have been harassed.

Women in medicine face the most frequent abuse. The report cites a 2017 survey which found that more than $40 \%$ of female medical students experienced sexual harassment from faculty or staff, compared to about $20 \%$ of female science students and $25 \%$ of female engineering students. Gender harassment, defined in the report as "sexist hostility and crude behavior," is the most common form

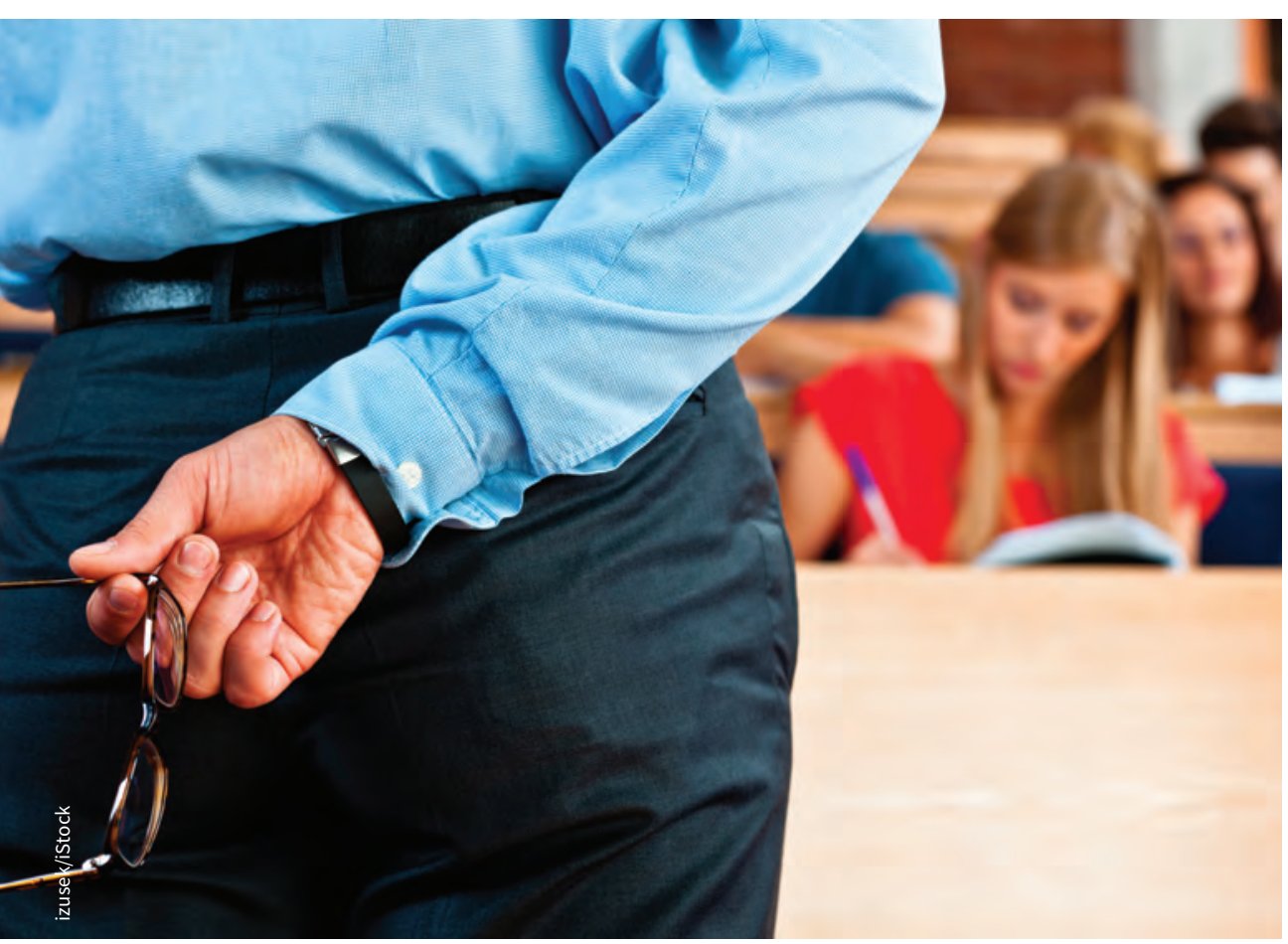

Rigid hierarchies and male domination compounds the problem of sexual harassment in scientific fields. women encounter. "As opposed to comeons, you can think of them as the putdowns," Johnson explained.

When severe and repeated, harassment can be as damaging as unwanted advances or pressure to trade sex for a career boost, the report said. It is most pervasive in medicine, where women face hostility and insults from both patients and colleagues.

"The cumulative effect of sexual harassment is significant damage to research integrity and a costly loss of talent," the report said. Women forgo career opportunities, leave their institutions and switch fields to avoid their harassers. Even men "don't stick around to watch their valued colleagues being disparaged," coauthor Lilia Cortina said at the report's release.

Many universities have created policies, reporting processes and training programs to combat harassment. But these tend to function to protect institutions from liability rather than to deter harassers or hold them accountable. "Unfortunately, academic institutions are often perceived as tolerant," the report's authors said.

The rigid hierarchies and male domination of scientific fields compound the problem, according to the report. "Most department chairs and deans are men. Most principal investigators are men. Most provosts and presidents are men." Although not all harassers are men, majority male leadership has resulted in "minimization, limited response, and failure to take the issue of sexual harassment or specific incidents seriously."

The report recommends system-wide changes to "move beyond legal compliance to address culture and climate," 
including using mentoring networks and committees to advise trainees and providing independent funding to diffuse the power of senior men over junior women.

Other recommendations include increasing the diversity of leadership, evaluating staff and faculty on professionalism and cooperation in hiring and promotion, improving supports for targets of harassment, and developing less formal ways of reporting abuse. Institutions should issue annual reports on their response to incidents. Consequences for harassment should be clear, escalating and punitive, instead of giving perpetrators only time off or fewer responsibilities.

"Academic institutions should consider sexual harassment equally important as research misconduct," and respond accordingly, the report concluded.

This may, however, be easier said than done. The National Academies of Sciences,
Engineering and Medicine have no codes of conduct and no way to expel harassers from their membership. In a recent tweet, Marcia McNutt, president of the National Academy of Sciences, said "Anyone who thinks it is easy has not tried to get a majority vote from an honorary society of more than 80 percent men over 70 years average age."

Lauren Vogel, CMAJ 